\title{
Multiplexed Five-Color Molecular Imaging of Cancer Cells and Tumor Tissues with Carbon Nanotube Raman Tags in the Near- Infrared
}

\author{
Zhuang Liu ${ }^{1, \dagger}$, Scott Tabakman ${ }^{2, \dagger}$, Sarah Sherlock ${ }^{2}$, Xiaolin $\mathrm{Li}^{2}$, Zhuo Chen ${ }^{2}$, Kaili Jiang ${ }^{3}$, Shoushan Fan ${ }^{3}$, and \\ Hongjie Dai ${ }^{2}(\bowtie)$ \\ ${ }^{1}$ Functional Nano \& Soft Materials Laboratory (FUNSOM), Jiangsu Key Laboratory for Carbon-Based Functional Materials \& Devices, \\ Soochow University, Suzhou 215123, China \\ ${ }^{2}$ Department of Chemistry, Stanford University, Stanford, CA 94305, USA \\ ${ }^{3}$ Department of Physics and Tsinghua-Foxconn Nanotechnology Research Center, Tsinghua University, Beijing 100084, China \\ Received: 30 November 2009 / Revised: 20 January 2010 / Accepted: 26 January 2010 \\ (C) The Author(s) 2010. This article is published with open access at Springerlink.com
}

\begin{abstract}
Single-walled carbon nanotubes (SWNTs) with five different C13/C12 isotope compositions and well-separated Raman peaks have been synthesized and conjugated to five targeting ligands in order to impart molecular specificity. Multiplexed Raman imaging of live cells has been carried out by highly specific staining of cells with a five-color mixture of SWNTs. Ex vivo multiplexed Raman imaging of tumor samples uncovers a surprising up-regulation of epidermal growth factor receptor (EGFR) on LS174T colon cancer cells from cell culture to in vivo tumor growth. This is the first time five-color multiplexed molecular imaging has been performed in the near-infrared (NIR) region under a single laser excitation. Near zero interfering background of imaging is achieved due to the sharp Raman peaks unique to nanotubes over the low, smooth autofluorescence background of biological species.
\end{abstract}

\section{KEYWORDS}

Carbon nanotubes, Raman scattering, biomedicine, multiplexed imaging

\section{Introduction}

Molecular imaging by optical techniques plays important roles in biology and medicine owing to high spatial resolution and detection sensitivity, providing not only geometrical but also functional information about biological and medical systems [1, 2]. However, various challenges remain in this area. Photo-bleaching is a problem for most organic fluorescent dyes, preventing long-term imaging and monitoring of fluorescently labeled substances [3]. High background due to autofluorescence of biological tissues also presents a problem in fluorescence imaging [4]. For in vivo imaging, the depth of tissue penetration by light is limited. The near-infrared (NIR) region from $700 \mathrm{~nm}$ to $1400 \mathrm{~nm}$ is an ideal window for optical imaging of biological systems due to the minimal tissue absorption and low autofluorescence in this

${ }^{\dagger}$ These authors contributed equally to the work.
Address correspondence to hdai@stanford.edu 
region [5].

Multi-color multiplexed molecular imaging is desired in order to simultaneously map out different species and reveal the geometrical and functional relations between different targets. Ideally, multi-color imaging of various molecular targets under a single excitation with both excitation and emissions in the narrow NIR range is desired. Although multi-color fluorescence imaging has been achieved using different fluorescent dyes or quantum dots with excitations/emissions in the visible to NIR range (400-900 nm) [6-8], imaging multiplicity is limited in the narrow NIR window due to spectral overlay between fluorescence peaks with full-width half-maximum (FWHM) as large as 50-100 nm. Also, autofluorescence, although low in the NIR region, still exists and varies between species in a biological sample [4]. Complex algorithms are needed to differentiate the background for fluorescence-based imaging [7].

Raman scattering emissions have narrow spectral features with FWHM within a few nm, allowing for increased imaging multiplicity [9]. Six or even more Raman "colors" have been obtained by conjugating different Raman active dyes on Ag or Au nanoparticles and simultaneously imaged on a substrate [10]. Threecolor non-specific Raman imaging under the skin of animals with NIR excitation and emissions has also been shown [11]. Thus far, multiplexed, multi-color (>3) targeted molecular Raman imaging of biological systems with high selectivity has not been achieved.

In recent years, carbon nanotubes have been explored in biological applications including molecular delivery [12-16], sensing and detection [17, 18], and imaging $[19,20]$. SWNTs are one-dimensional materials [21] with unique intrinsic optical properties such as photoluminescence in the NIR $[19,22]$ and strong resonant Raman signatures [23], both of which are useful for biological detection and imaging. We have shown that SWNTs with three different C13/C12 isotope compositions exhibit shifted Raman G-band peaks, and can be used for three-color multiplexed Raman imaging of live cells [24].

In this work, five SWNT samples with various C13/C12 ratios were synthesized, functionalized, and conjugated with different targeting ligands for specific labeling and imaging of biological samples. Relative protein expression levels on several human cancer cell lines were analyzed. Five-color multiplexed Raman imaging was carried out for cancer cells in culture and in tumors ex vivo. We observed tumor vasculatures labeled by one SWNT color and cancer cells by other colors. Unexpectedly, we observed a drastic upregulation of epidermal growth factor receptor (EGFR/Her1) expression on LS174T human colon cancer cells during tumor growth in nude mice (not present in vitro in cell culture). This is the first multicolor molecular Raman imaging of five different biological targets with excitation/emission in the NIR region under a single laser excitation, with essentially zero interfering background.

\section{Results and discussion}

\subsection{Five unique, target specific SWNT Raman scattering labels}

Isotopically modified SWNTs were synthesized by using mixtures of $\mathrm{C} 13$ methane and $\mathrm{C} 12$ methane in chemical vapor deposition (CVD) growth of nanotubes $[24,25]$. SWNT samples grown with gas phase C13 ratios of $100 \%, 65 \%, 50 \%, 25 \%$, and $0 \%$ exhibited Raman G-band peaks at $1529 \mathrm{~cm}^{-1}, 1546 \mathrm{~cm}^{-1}, 1559 \mathrm{~cm}^{-1}$, $1575 \mathrm{~cm}^{-1}$, and $1590 \mathrm{~cm}^{-1}$, which are defined as Color1, Color2, Color3, Color4, and Color5, respectively (Fig. 1). As grown SWNTs were purified as reported earlier [25]. Phospholipid-polyethylene glycol-amine (DSPEPEG5000- $\mathrm{NH}_{2} / \mathrm{PL}-\mathrm{PEG5000-Amine)} \mathrm{was} \mathrm{used} \mathrm{to}$ functionalize SWNTs and afford water soluble SWNTs (length 50-300 nm) after centrifugation to remove large bundles and filtration to remove excess surfactant [24, 26-28]. The five functionalized SWNT samples were conjugated to five different targeting antibodies and a peptide, including Erbitux (anti-EGFR/Her1) to target EGFR/Her1, cyclic arginine-glycine-aspartic acid (RGD, cyclo-RGDyK) peptide to target integrins such as $\alpha v \beta 3$, goat anti-human carcinoembryonic antigen (CEA) antibody to recognize CEA, Rituxan (anti-CD20) to target CD20 protein, and Herceptin (anti-Her2) to target Her2/neu protein (Fig. 1(a)) $[19,24]$. 

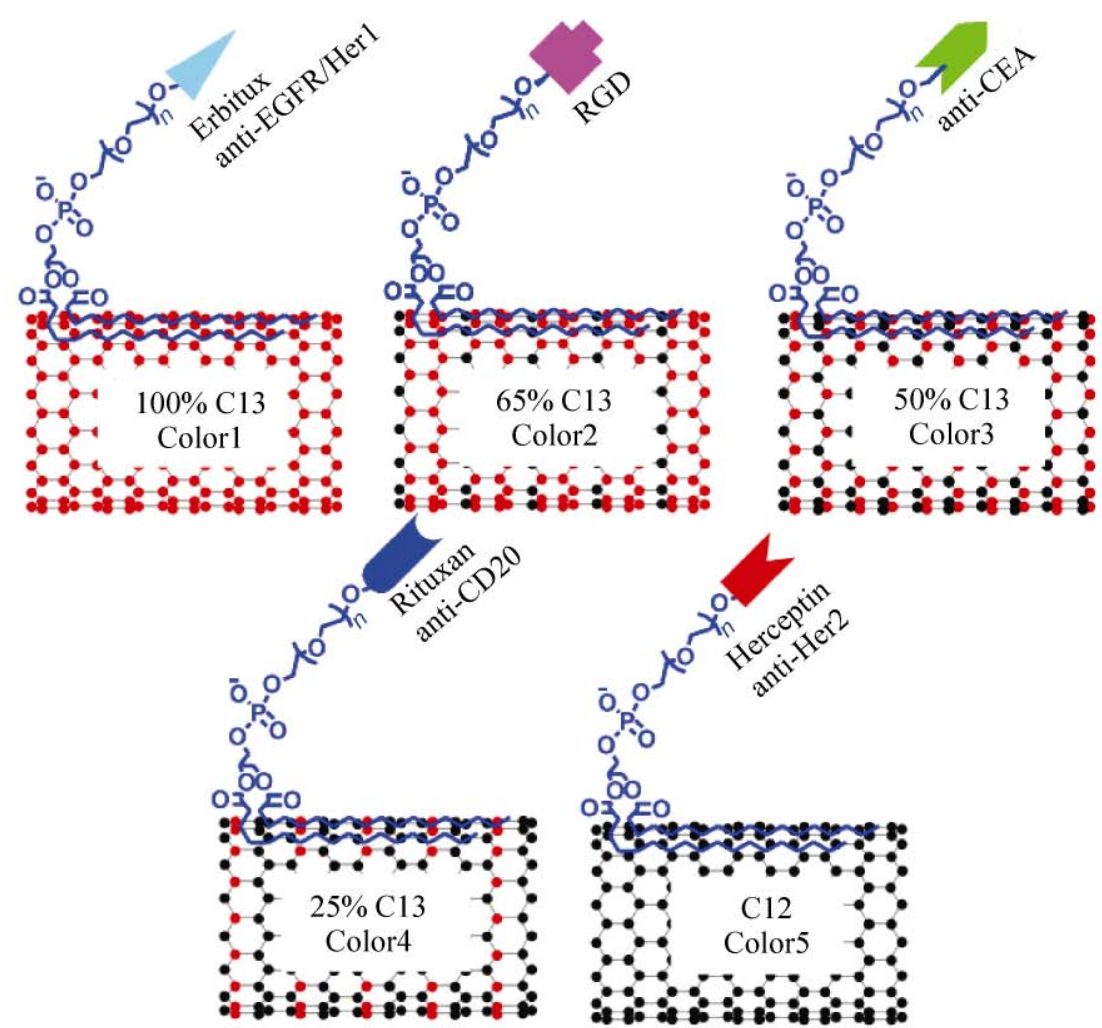

(a)

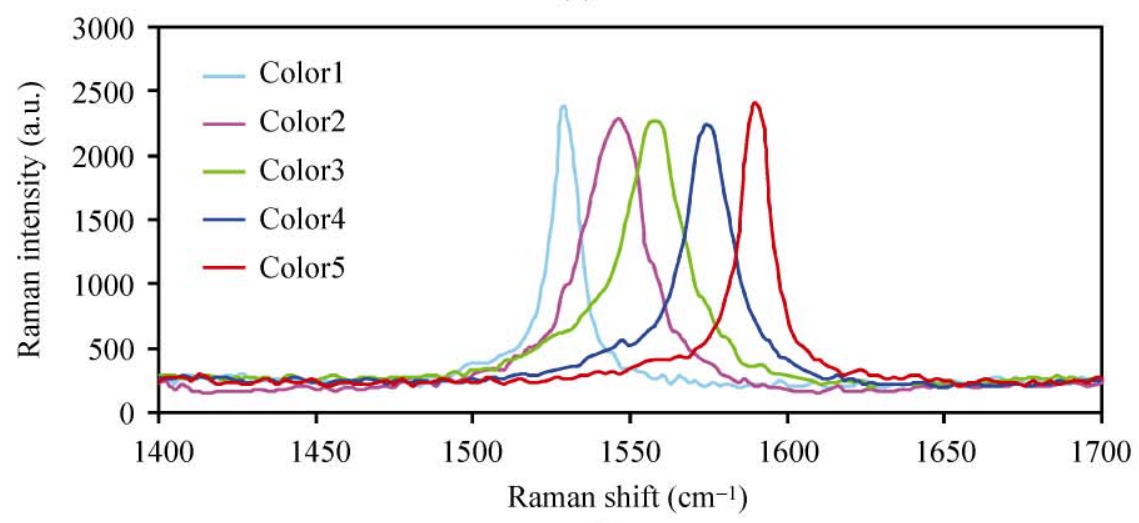

(b)

Figure 1 SWNTs with different Raman "colors". (a) Scheme of isotopically modified SWNTs, grown from FeRu catalysts, conjugated with different targeting ligands. Color1, 2, 3, 4, and 5 represent FeRu-grown SWNTs with C13 percentages (determined by the C13-methane/C12-methane growth gas ratios) of $100 \%, 65 \%, 50 \%, 25 \%$, and $0 \%$, respectively. (b) Raman spectra of the five different SWNT samples in aqueous solutions. The shift of SWNT Raman G-band peak is clearly dependent on the $\mathrm{C} 13 / \mathrm{C} 12$ ratio in the SWNTs. The average shift between two adjacent colors is about $15 \mathrm{~cm}^{-1}$

\subsection{Five-plexed in vitro Raman imaging and semi- quantitative analysis of cancer cell surface biomarkers}

We carried out multiplexed two-color cell imaging of Her1 and Her2, two of the Her-family antigens on various cancer cells. Six different human cancer cell lines were incubated with a mixture of Color1-antiEGFR/Her1 and Color5-antiHer2 at $4{ }^{\circ} \mathrm{C}$ for $1 \mathrm{~h}$ and then washed by phosphate buffered saline (PBS). Spectroscopic mapping of cells over a large area was then carried out. The smooth autofluorescence background from $1470 \mathrm{~cm}^{-1}$ to $1640 \mathrm{~cm}^{-1}$ was subtracted to reveal pure SWNT G-band Raman peaks (see Figs. S-1 and S-5 in the Electronic Supplementary Material (ESM)). The $G$ peak intensities of C13 and C12 SWNTs at each location were then integrated and used to construct two-color Raman images (Fig. 2(a)). Strong Color1 signals (assigned to red) but not Color5 (green) were observed from BT20 and MDA-MB-468 cells, suggesting high Her1, but low Her2 expressions on these cells (Fig. 2(a), 2nd and 6th rows). MDA-MB-231 cells also showed moderate expression of EGFR/Her1 (Fig. 2(a), 3rd row). On the other hand, high Her2 expression was noted on BT474 and MDA-MB-361 cells as evidenced by the observed strong Color5 (Her2) signals on those cells (Fig. 2(a), 1st and 4th rows). Other cell lines exhibited low Her2 expression (Fig. 2(a)).

Since the SWNT concentrations used in the staining solution were far beyond the saturation levels under our experimental conditions (see Fig. S-3 in the ESM), 


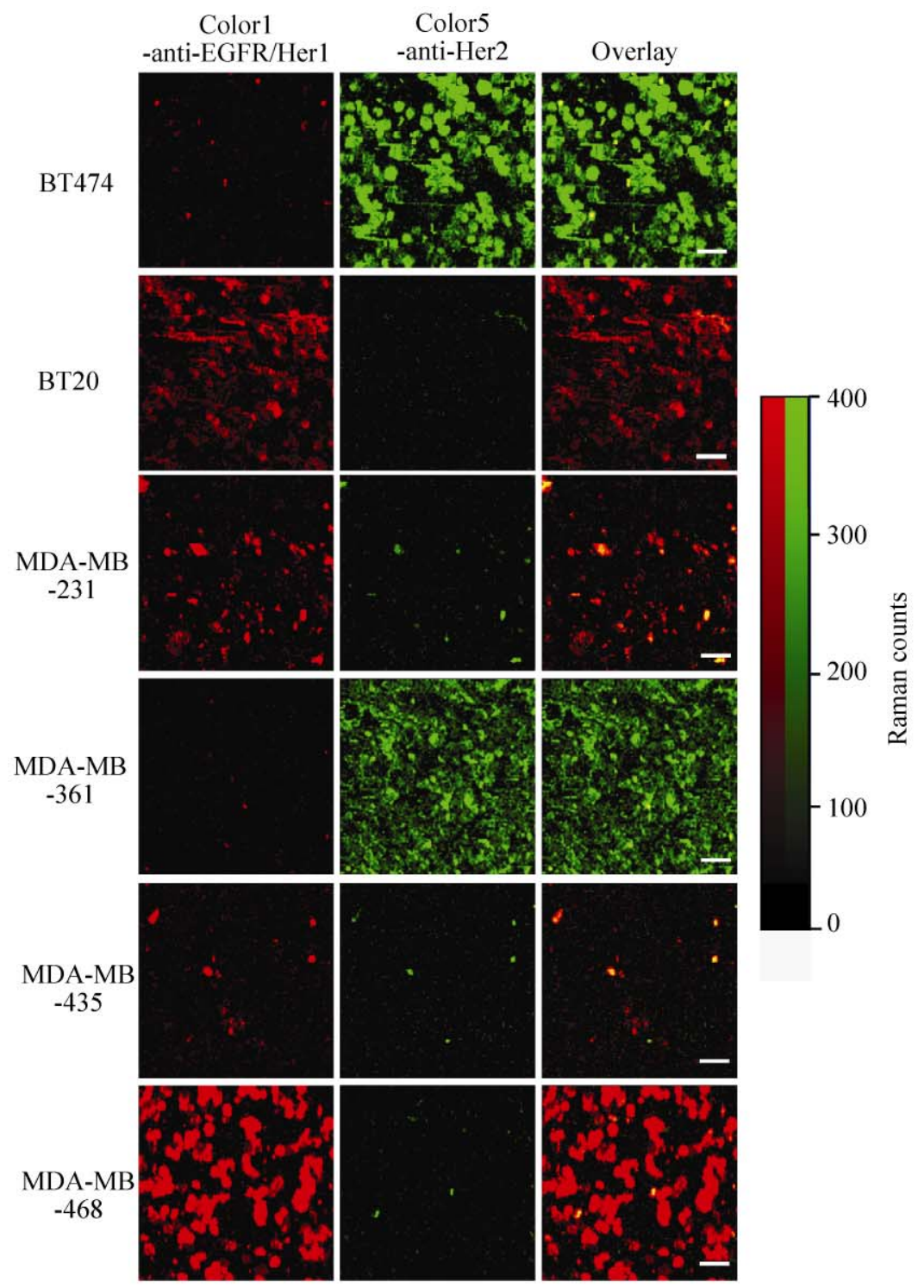

(a)

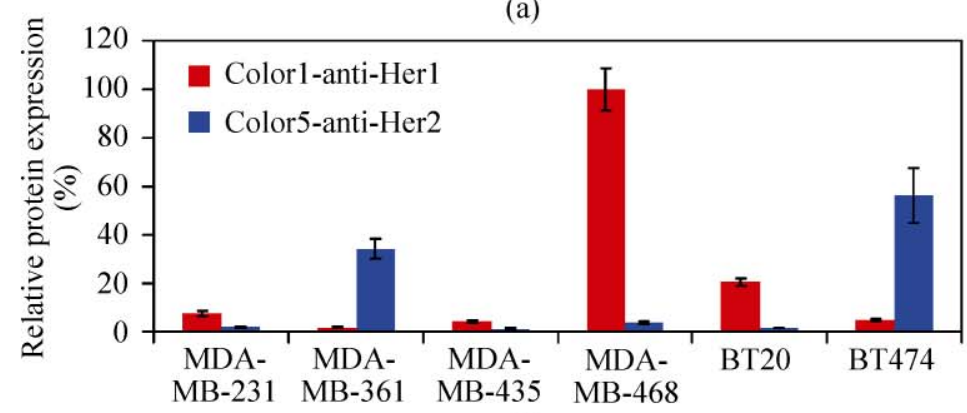

(b)

Figure 2 Two-color Raman imaging of Her1 and Her2 of different breast cancer cell lines. (a) Two-color Raman images of six breast cancer cell lines. Cells were stained by a mixture of Color1-anti-EGFR/Her1 and Color5-anti-Her2 conjugates for Raman spectroscopic imaging. 150-400 cells were imaged in each mapping field. Scale bar $=50 \mu \mathrm{m}$. (b) Semiquantitative analysis of two-color Raman imaging data. Averaged Raman intensity per cell of each breast cancer cell line was calculated by dividing the sum of Raman intensity in each spectroscopic image by the total number of cells in the mapping area counted from the related optical image (see Methods section), and normalized by Raman scattering intensity factors of two types of SWNTs (Fig. S-2 in the ESM). Relative EGFR/Her1 and Her2 expression levels were determined by the average Raman intensities of Color 1 and Color 5 per cell, respectively. Error bars were based on triplicated images 
each cell surface receptor should have bound an antibody-conjugated nanotube, and because the Raman intensity of SWNTs is linearly dependent on the SWNT concentration, the averaged Raman intensity per cell is proportional to the mean receptor level on cells, which allowed us to quantify relative protein expression levels of different cell lines. The EGFR/Her1 expression levels followed a trend of MDA-MB-468 > BT20 > MDA-MB-231 > MDA-MB-435 > BT474 MDA-MB-361. In the case of Her2, we observed BT474 $>$ MDA-MB$361>$ BT20 MDA-MB-231 MDA-MB-468 MDAMB-435 (Fig. 2(b)). These trends are consistent with flow cytometry data with fluorescently labeled antibodies (see Fig. S-4 in the ESM), as well as previous report [29], suggesting that semiquantitative measurement of surface protein expression levels can be achieved by SWNT-based labeling and Raman spectroscopic imaging.

Next, we used five-plexed SWNT Raman tags to label various cancer cells over-expressing different receptors. After incubation with the five-color SWNT mixture (Color1-anti-EGFR/Her1, Color2-RGD, Color3-antiCEA, Color4-anti-CD20, Color5-anti-Her2), cells were washed by PBS and subjected to Raman spectroscopic imaging. The Raman spectrum at each pixel was deconvoluted to those of the five Raman colors after background subtraction (see Methods section for detailed data processing), affording five-color Raman images, with relative Raman intensities of deconvoluted spectra at each pixel (Fig. 3). The five cancer cell lines with different surface protein expression profiles were successfully labeled by SWNT tags with high specificity, showing distinct Raman "colors" on MDA-MB-468, U87MG, LS174T, Raji, and BT474 cells, respectively (Fig. 3, top 5 rows). Minimal crosstalk between different colors was seen by analysis of the spectra. The non-specific binding (NSB) of mismatched nanotube conjugates on cells was negligible (positive binding over NSB signals = 50:1-100:1). When the five cell lines were mixed together and stained by a mixture of five-color SWNT conjugates, Raman spectroscopic imaging clearly differentiated five different types of cancer cells by their respective SWNT Raman labels with minimal overlapping of colors between cell types. Five-color multiplexed molecular Raman imaging of cells in vitro is thus achieved by SWNT Raman labels.

\subsection{Molecular imaging of ex vivo tumor slices via five-plexed SWNT Raman labels}

To explore the utility of multi-color SWNT Raman tags for probing samples with high biological and medical relevance, we used the five-plexed SWNTs for ex vivo tumor staining and Raman imaging, aimed at detecting multiple species related to cancer in a single experiment, and resolving the locations and spatial relations of various molecular targets in the tumor. Nude mice bearing LS174T human color cancer tumors were sacrificed 15 days after tumor inoculation. The collected tumors were frozen-sliced into $5 \mu \mathrm{m}$ thick tumor slices and stained with the five-color, five-plexed SWNT mixture (see Methods section). Raman spectroscopic mapping of the slices was then performed at both high resolution over a small area and low resolution over the entire slice (Figs. 4(b) and $4(\mathrm{c})$, respectively), yielding multi-color Raman images of protein expression. As expected from the in vitro cell imaging results (Fig. 3), cancer cells in LS174T tumor slices showed a high level of CEA expression (Fig. 4, blue color). Tumor vessel-like structures were positively stained by Color5-RGD conjugates, indicating high integrin expression on the tumor vasculature, most likely the integrin $\alpha \mathrm{v} \beta 3[30,31]$ (Fig. 4, red color). It is well known that integrin $\alpha v \beta 3$ receptor regulates tumor angiogenesis and is overexpressed on various tumor vasculature endothelial cells in vivo $[32,33]$.

Interestingly, we found that EGFR/Her1 surface receptor, which had low expression in vitro on LS174T cells [34, 35], was highly up-regulated in vivo in the xenograft tumor probed 15 days post inoculation, and was clearly stained by Color1-anti-EGFR/Her1 (Fig. 4). The high degree of overlay of Color1 (yellow, EGFR/Her1) and Color3 (blue, CEA) in the Raman images (Figs. 4(b) and 4(c)) suggested colocalization of EGFR/Her1 and CEA over-expression on LS174T cancer cells, with the former developed in vivo. In addition, the LS174T tumor slice was also diffusely stained by Color2-anti-Her2, suggesting a moderate up-regulation of Her2 receptor in the xenograft LS174T tumor model. In the literature, high tumor uptake of 


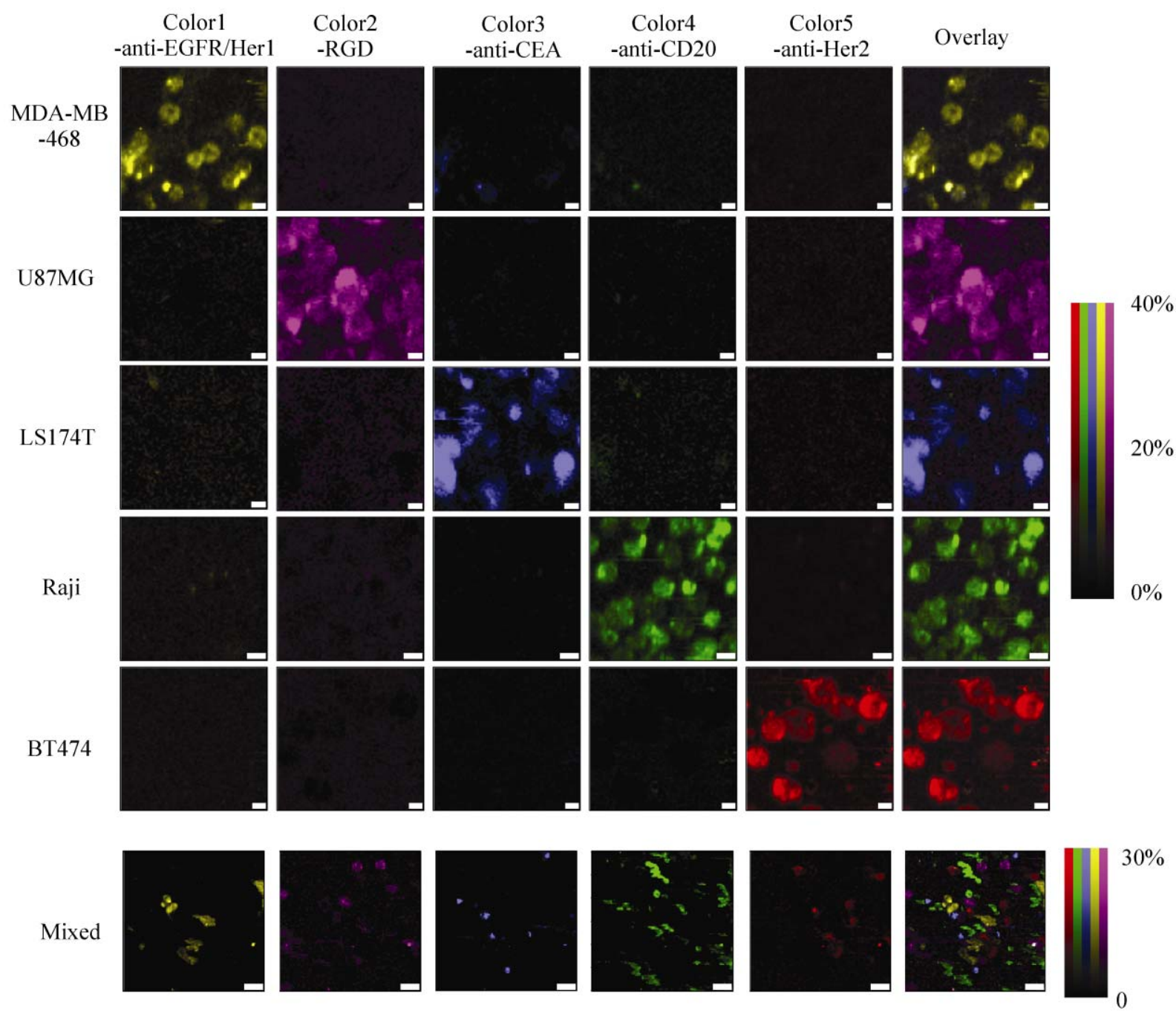

Figure 3 Five-color Raman imaging of cancer cells. In the top 5 rows (scale bars $=10 \mu \mathrm{m}$ ), each cell line was stained by a five-color multiplexed SWNT mixture for Raman spectroscopic imaging. In the bottom row (scale bars $=40 \mu \mathrm{m}$ ), 5 types of cancer cells were mixed together and the incubated with the five-color SWNT mixture. Spectral deconvolution was used to resolve different colors of SWNTs (see ESM). The colors are scaled as the percentage of the maximum relative Raman intensity of the deconvoluted spectra (the most intense spectrum of any color is assigned 100\%). Spectra of five SWNT solutions at the same optical density (OD) as the excitation wavelength were inputted to deconvolute the raw data (Fig. S-2 in the ESM)

anti-EGFR/Her1 antibody in the LS174T xenograft mouse model through an in vivo biodistribution study has only been reported in one recent work, despite the low EGFR/Her1 expression level on LS174 cells in vitro [35]. Similar observations have been made for low levels of Her2 in the same tumor model [36]. However, no prior imaging result exists to show the spatial relationship of CEA and EGFR expression in this xenograft tumor model. Our finding suggests that the expression profile of cancer cell receptors could change from cell culture to tumor growth in vivo. The over-expressions of EGFR and Her2, which play important roles in the process of cancer cell proliferation, is associated with many types of cancers and has become one of the most important targets in cancer therapy [37]. Our observation suggests the importance of in vivo and ex vivo probing and evaluation of EGFR for both diagnostic and targeted treatment proposes. It also highlights the usefulness of multiplexed imaging to probes multiple targets 


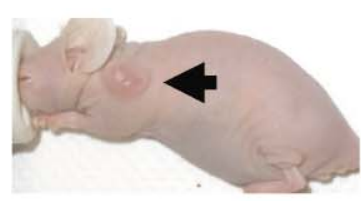

(a)

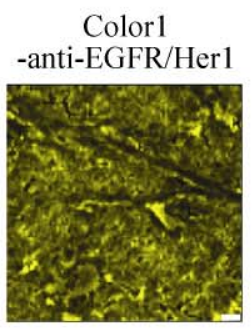

Color4 -anti-CD20

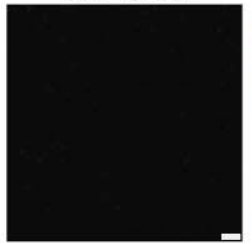

Color 1 -anti-EGFR/Her 1

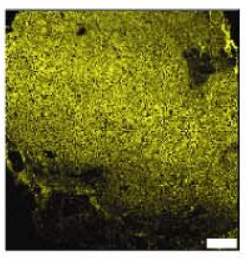

Color 4 -anti-CD20

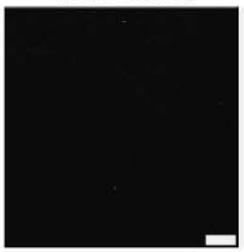

Color 2 -anti-Her2

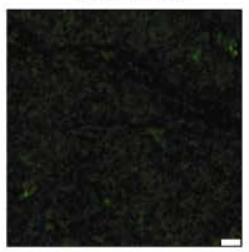

Color5 -RGD

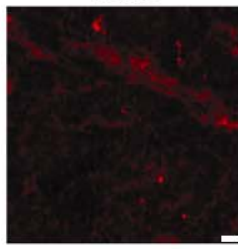

(b)

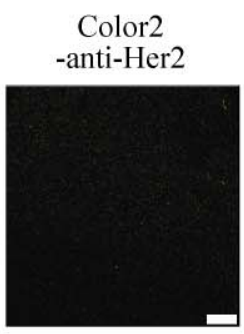

Color5 -RGD

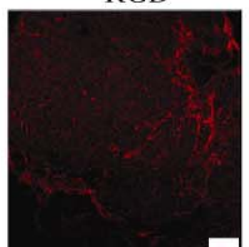

Color3 anti-CEA

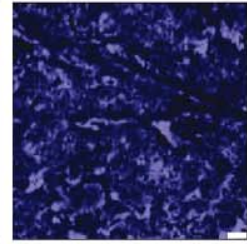

Overlay

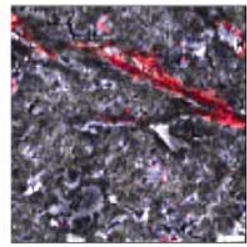

Color3 anti-CEA

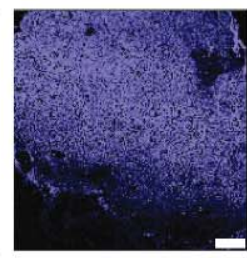

Overlay

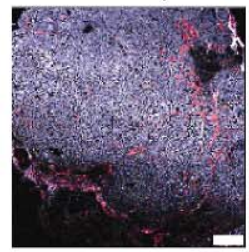

Bright field

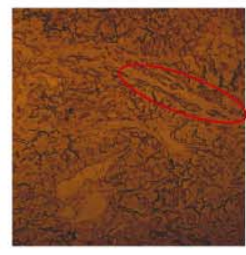

Bright field
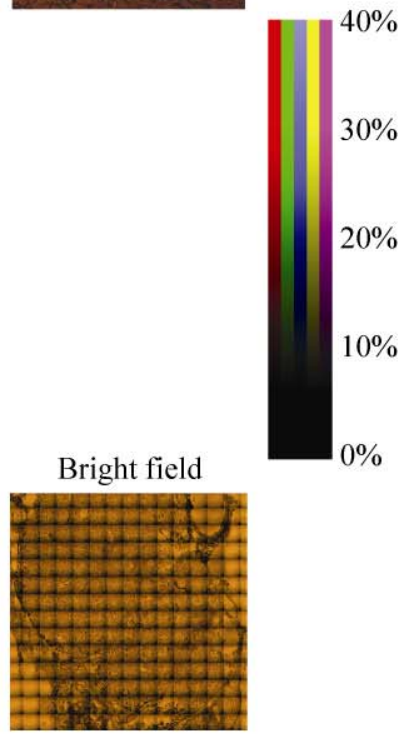

(c)

Figure 4 Multi-color ex vivo tumor imaging by Raman imaging. Note that SWNT conjugates used here were different from those used in the previous cell imaging experiments. Hipco SWNTs (C12) with a greater Raman scattering intensity factor were used as Color5. (a) A photo of a LS174T tumor-bearing nude mouse used in this study. The arrow is pointing to a tumor. (b) Five-color Raman images of a LS174T human colon tumor slice from a mouse model. Separate images representing five protein expression levels were generated by deconvoluting the raw spectra at each pixel into fractional percentages of the five intensity factor-corrected, G-band spectra. Colors are plotted as a percentage of maximum intensity (the most intense pixel of any color is assigned 100\%). The overlay image exemplifies the high degree of correlation between CEA expression and EGFR expression. Note that in overlay images Color5-RGD intensity is doubled for clarity. LS174T cells in the tumor showed high levels of EGFR/Her1 (yellow) and CEA (blue) receptors, and colocalization. Integrin expression visualized by Color5-RGD Raman staining (red) appeared to be on the tumor vessels. A relatively low level of Her2 expression was also observed on LS174T tumor cells. Right: a corresponding bright field optical image of the tumor slice. The highlighted area is a tumor vessel. Integrin expression, likely that of $\alpha \mathrm{v} \beta 3$, was clearly associated with blood vessels in the tumor. Scale bar $=50 \mu \mathrm{m}$. (c) Large area whole tumor slice five-color Raman images. Five separate images for each protein expression level were generated as in Fig. 4(b), and are accompanied by an overlay image, in which Color5-RGD intensity has been doubled for clarity. The bright field image of the whole tumor slice was obtained by stacking multiple small area images taken under a $10 \times$ objective. Blood vessels going through the tumor are clearly visualized in the Color5 image. Scale bar $=1 \mathrm{~mm}$ 
with high efficiency and spatial resolutions to glean valuable insights into the complexities of molecular pathways and networks in tumors.

\section{Conclusions}

Five-color multiplexed imaging in the narrow NIR window has not been achieved by fluorescence imaging using either organic dyes or quantum dots owing to the wide fluorescence spectra relative to the narrow NIR window. In our Raman imaging, excitation $(785 \mathrm{~nm})$ and scattered photons (892-897 $\mathrm{nm}$ ) are all in the narrow NIR window with low tissue absorption and autofluorescence background (see Fig. S-5 in the ESM). SWNTs have a single, narrow Raman G-band peak with FWHM $<2 \mathrm{~nm}$, allowing for high degrees of multiplicity. As many as five Raman colors are simultaneously imaged under a single laser excitation for measuring five different targets in a single Raman mapping scan. Although previous proof-of-concept work has shown that Raman tags based on molecules and metal nanoparticle complexes can be used for multiplexed imaging with many colors $[10,11]$, our work is the first to use multiple ( $>3)$ Raman colors to successfully label different biological species with specificity to afford multiplexed molecular imaging. Nanotube Raman tags are advantageous over common Raman dye molecules, such as cyanine dyes and rhodamine derivatives, as they present simple yet intense spectra, without the complex, multiple Raman peaks and high fluorescence emission background of the latter. The simple NIR Raman scattering spectra of SWNTs allow for facile spectral deconvolution without the need of sophisticated algorithms, including those necessary to map out sample autofluorescence, which varies in space over biological samples [4,7]. Also, important is that nanotube Raman signals exhibit no quenching or bleaching under a wide range of imaging conditions [38].

The sharp single G-peak feature in the SWNT Raman spectrum enables us to easily distinguish SWNT Raman signals from the low and slowly varying autofluorescence background, allowing for "background-free" optical imaging with high imaging and detection sensitivities (see Fig. S-1 in the ESM). On the other hand, the Raman spectral acquisition times reported in this manuscript are substantially longer than those used in confocal fluorescence microscopy, which represents a practical limitation of the approach. Promising nonlinear Raman measurement techniques [39] have recently been reported which may potentially reduce acquisition time and improve the utility of multiplexed SWNT Raman labels.

Besides utilizing the isotope-dependent Raman G peak shift, the SWNT radial breathing mode (RBM) peak position exhibits a systematic diameter dependence [23]. Even more Raman colors could be achieved for chirality/diameter separated SWNTs [40]. Taken together, SWNTs are promising highperformance NIR Raman tags for multi-color multiplexed optical imaging and detection in vitro, ex vivo and potentially in vivo.

\section{Methods}

\subsection{Synthesis of isotopically modified SWNTs}

The synthesis of isotopically modified SWNTs was performed on a silica supported $\mathrm{Fe}-\mathrm{Ru}$ bimetallic catalyst using $\mathrm{CH}_{4} \mathrm{CVD}$ growth following our previously reported protocol at $850^{\circ} \mathrm{C}$ [25]. To control isotope compositions of SWNTs, $\mathrm{C} 12 \mathrm{CH}_{4}$ gas and $\mathrm{C} 13$ $\mathrm{CH}_{4}$ gas (99\% pure) were mixed at desired ratios by flow controllers during SWNT growth. The as-grown product was etched in $15 \%$ HF solution in water $(75 \%)$ and ethanol (10\%) overnight at room temperature to remove the silica support. The purified SWNTs were then washed thoroughly with ethanol and distilled water to neutral $\mathrm{pH}$ and stored for further use. Note that pure $\mathrm{C} 12 \mathrm{Fe}-\mathrm{Ru}$ SWNTs were used in cell incubation and imaging (Figs. 2 and 3) while Hipco SWNTs (pure C12) from Carbon Nanotechnology Inc. were used as Color5 (Color5-RGD) in tumor slice staining and imaging (Fig. 4).

\subsection{Functionalization and bioconjugation}

Functionalization and bioconjugation of SWNTs have been well established in our previous reports $[14,19$, 27, 28]. DSPE-PEG5000- $\mathrm{NH}_{2}$ (NOF Corporation) at a concentration of $1 \mathrm{mg} / \mathrm{mL}$ was sonicated in distilled water for $1 \mathrm{~h}$ with SWNTs prepared as above. The water bath was changed every $20 \mathrm{~min}$ to avoid over-heating. 
The nanotube suspension was then centrifuged at $24000 \mathrm{~g}$ for $6 \mathrm{~h}$ to remove aggregates and metal catalysts and collect the supernatant. Excess DSPE-PEG5000$\mathrm{NH}_{2}$ was removed by filtration through $100 \mathrm{kDa}$ MWCO ultracentifugal filter devices (Millipore Amicon Ultra) prior to conjugation to antibody or peptide.

Erbitux (anti-Her1), Rituxan (anti-CD20), and Herceptin (anti-Her2) were gifts from Drs. Dean Felsher and Sanjiv Gambhir (Stanford University Medical Center, Stanford, CA, USA). Polyclonal goat anti-CEA antibody was purchased from BiosPacific Inc. (Emeryville, CA, USA). These antibodies were thiolated by mixing them with 2-iminothiolane hydrochloride (Traut's reagent, Pierce) at 1:10 molar ratio (all IgG masses assumed to be $150 \mathrm{kDa}$ ) in PBS in the presence of $20 \mathrm{mmol} / \mathrm{L}$ EDTA for $2 \mathrm{~h}$. Unreacted Traut's reagent was removed by filtration through 100 kDa MWCO ultracentrifugal filter devices. The thiolated antibodies were used immediately in the following conjugation. Thiolated RGD (RGD-SH) was a gift from Dr. Xiaoyuan Chen, (NIBIB/NIH, Bethesda, MD, USA) and used directly without further modification.

Functionalized SWNTs at concentrations of $300 \mathrm{nmol} / \mathrm{L}$ (calculated by extinction at $808 \mathrm{~nm}$ [37], $\left.\varepsilon_{808 \mathrm{~nm}}=0.0079(\mathrm{nmol} / \mathrm{L})^{-1} \cdot \mathrm{cm}^{-1}\right)$ were reacted with $1 \mathrm{mmol} / \mathrm{L}$ sulfosuccinimidyl [4- $\mathrm{N}$-maleimidomethyl] cyclohexane-1-carboxylate (sulfo-SMCC, Pierce) for $2 \mathrm{~h}$ in PBS at $\mathrm{pH}$ 7.4. After thorough removal of excess sulfo-SMCC by filtration through $100 \mathrm{kDa}$ MWCO ultracentrifugal filter devices, the SWNT solutions were mixed with thiolated antibodies in PBS in the presence of $5 \mathrm{mmol} / \mathrm{L}$ EDTA. In the reaction buffer, the final SWNT concentration was $300 \mathrm{nmol} / \mathrm{L}$, while that of antibodies was $1.5 \mu \mathrm{mol} / \mathrm{L}(\sim 1: 5$ molar ratio). The reactions were allowed to proceed overnight at $4{ }^{\circ} \mathrm{C}$, affording SWNT-antibody conjugates used for cell incubation and imaging. RGD-SH was conjugated to SWNTs using a similar protocol except that $100 \mu \mathrm{mol} / \mathrm{L}$ of RGD-SH was used in the reaction mixture. $5 \mathrm{mmol} / \mathrm{L}$ tris(2-carboxyethyl)phosphine hydrochloride(TCEP) was added to prevent the oxidation of the thiols.

\subsection{Cell culture and incubation}

BT20, BT474, MDA-MB-231, MDA-MB-361, and MDAMB-468 human breast cancer cell lines, MDA-MB-435
M14 melanoma cell line, U87MG brain cancer cell line, LS174T human colon cancer cell line, and Raji human B-cell lymphoma cell line were either obtained from the American Type Culture Collection (ATCC) or gifts from Dr. Xiaoyuan Chen (NIBIB/NIH, Bethesda, MD, UDA) and Dr. Dean Felsher (Stanford University Medical Center, Stanford, CA, USA). Descriptions of cell propagation and subculturing conditions may be found in the ESM.

For cell incubation, $200 \mu \mathrm{L}$ of cells $\left(\sim 1 \times 10^{6}\right)$ were incubated with $50 \mu \mathrm{L}$ of SWNT solution (con- taining either a two-color or five-color SWNT mixture) in PBS for $1 \mathrm{~h}$ at $4{ }^{\circ} \mathrm{C}$. The final concentration of each color of SWNT (average length $\sim 150 \mathrm{~nm}$ ) [41] in the incubation solution was $10 \mathrm{nmol} / \mathrm{L}$. Cells were washed with PBS three times before Raman imaging.

\subsection{Raman imaging}

Confocal Raman spectroscopic imaging was carried out using a HORIBA-Jobin-Yvon Raman confocal microscope with a $785 \mathrm{~nm}$ laser $(80 \mathrm{~mW})$ as the excitation light source. A drop of cell suspension was sealed between two thin plastic cover-slides for imaging. A $50 \times$ objective was used with $\sim 1 \mu \mathrm{m}$ laser spot size. A $1 \mathrm{~mm}$ pinhole was applied to afford a spatial resolution of $\sim 1 \mu \mathrm{m}$ in $x y z$-axes. Each Raman spectroscopic map contains at least $100 \times 100$ spectra with $0.5 \mathrm{~s}$ integration time for each spectrum. $2 \mu \mathrm{m}$ and $1 \mu \mathrm{m}$ step sizes were used for Raman mapping to obtain large area images (Fig. 2, last row of Fig. 3, and Fig. 4(b)) and zoomed-in images (Fig. 3, top five rows), respectively. $40 \mu \mathrm{m}$ steps were used for whole tissue slice mapping (Fig. 4(c)).

\subsection{Background subtraction}

The autofluorescence background in the Raman spectrum of cell or tissue images was low and smooth in the range of interest from $1470 \mathrm{~cm}^{-1}$ to $1640 \mathrm{~cm}^{-1}$, which is the Raman G-band region of SWNTs. We simply subtracted a line from $1470 \mathrm{~cm}^{-1}$ to $1640 \mathrm{~cm}^{-1}$ as to remove the smooth background at each pixel (Fig. S-1 in the ESM), producing essentially background-free spectra used for Raman image construction and data analysis. A typical spectrum of positively stained cells by SWNTs exhibits 1000-5000 G-band Raman counts, 
while the standard deviation of background noise in the spectrum of non-treated cells is $20-40$. The signal to noise ratio is $\sim 100$ at each pixel.

\subsection{Data analysis for two color Raman imaging}

Raman spectra of Color1 (pure C13) and Color5 (pure C12) have little overlay between each other. Intensities of individual peaks (1510-1550 $\mathrm{cm}^{-1}$ for Color1, 1570 $1610 \mathrm{~cm}^{-1}$ for Color5) were integrated independently at each pixel to construct the two-color images shown in Fig. 2. Red and green colors were artificially assigned to Color1 and Color5 SWNT signals, respectively.

For semiquantitative analysis of relative protein expression levels, all spectra in each spectroscopic map (Fig. 2(a)) were summed. The G-band peak height in the sum spectrum was determined as the total Raman counts for the whole map. The total number of cells in the mapping area was counted from the corresponding optical bright field image, and used to calculate an averaged Raman counts per cell, which was used to determine the relative protein level on cells after normalization of SWNT Raman scattering intensity factor (see Fig. S-2 in the ESM). Raman scattering intensity factors of the various SWNT materials were determined empirically by normalizing suspension absorbances at the excitation wavelength $(785 \mathrm{~nm})$, and were linearly proportional to SWNT concentration, determined by UV-vis-NIR absorbance.

\subsection{Data analysis for five-color Raman imaging}

To create a five-color image based upon relative scattering intensities, the spectra of five pure SWNT solutions (after background subtraction) at the same OD at $785 \mathrm{~nm}$ (Fig. S-2 in the ESM) were used for deconvolution of a background-subtracted, recorded spectrum, to obtain five Raman G peak components at each mapping site, thus accounting for the Raman scattering intensity factor of each SWNT material. The software generated relative Raman intensity values (the deconvoluted intensity divided by the input spectral intensity) of five colors at each pixel. A color scale was then assigned to each of the five peaks corresponding to the "percentage of the maximum" deconvoluted intensity of the entire image $(100 \%$ represents the intensity of the strongest pixel among all 5 colors in an image). Separate and overlaid fivecolor Raman images were then constructed.

\subsection{Tumor inoculation, slicing, and staining}

Athymic nude mice obtained from Harlan Sprague Dawley Inc. were housed in Stanford Research Animal Facility (RAF) under Stanford Institutional Animal Care and Use Committee (IACUC) protocols. Approximately $5 \times 10^{6}$ LS174T cells were implanted subcutaneously on the shoulder of nude mice. Mice were sacrificed 15 days after tumor inoculation. Tumors taken from the mice were immediately placed into optimal cutting temperature (OCT) medium and frozen by dry ice. $5-\mu \mathrm{m}$ thick tumor slices were cut by a microtome, placed on quartz slides, and fixed with dry ice-cold acetone. After blocking with 3\% fetal bovine serum (FBS) and 0.1\% Tween-20 in PBS for 30 $\mathrm{min}$ at room temperature, tissue slides were incubated with a solution of five-color SWNT mixture in RMPI-1640 cell medium with $10 \%$ FBS for $1 \mathrm{~h}$. The total SWNT concentration in the incubation solution was $20 \mathrm{nmol} / \mathrm{L}(4 \mathrm{nmol} / \mathrm{L}$ for each color of SWNT conjugate). These tissue slides were then washed 3 times with PBS (5 min each time) and then briefly with distilled water. Slides were dried in air for $30 \mathrm{~min}$ before Raman spectroscopic imaging.

\section{Acknowledgements}

This work was supported partially by CCNE-TR at Stanford University, NIH-NCI R01 CA135109-02, and Ensysce Biosciences Inc.

Electronic Supplementary Material: Supplementary material (details of cell culture procedure and five additional figures) are available in the online version of this article at http://dx.doi.org/10.1007/s12274-0101025-1 and is accessible free of charge.

Open Access: This article is distributed under the terms of the Creative Commons Attribution Noncommercial License which permits any noncommercial use, distribution, and reproduction in any medium, provided the original author(s) and source are credited. 


\section{References}

[1] Massoud, T. F.; Gambhir, S. S. Molecular imaging in living subjects: Seeing fundamental biological processes in a new light. Gene. Dev. 2003, 17, 545-580.

[2] Wagnieres, G. A.; Star, W. M.; Wilson, B. C. In vivo fluorescence spectroscopy and imaging for oncological applications. Photochem. Photobiol. 1998, 68, 603-632.

[3] Song, L. L.; Hennink, E. J.; Young, I. T.; Tanke, H. J. Photobleaching kinetics of fluorescein in quantitative fluorescence microscopy. Biophys. J. 1995, 68, 2588-2600.

[4] Aubin, J. E. Autofluorescence of viable cultured mammaliancells. J. Histochem. Cytochem. 1979, 27, 36-43.

[5] Frangioni, J. V. In vivo near-infrared fluorescence imaging. Curr. Opin. Chem. Biol. 2003, 7, 626-634.

[6] Alivisatos, A. P.; Gu, W. W.; Larabell, C. Quantum dots as cellular probes. Ann. Rev. Biomed. Eng. 2005, 7, 55-76.

[7] Xing, Y.; Chaudry, Q.; Shen, C.; Kong, K. Y.; Zhau, H. E.; Chung, L. W.; Petros, J. A.; O'Regan, R. M.; Yezhelyev, M. V.; Simons, J. W. et al. Bioconjugated quantum dots for multiplexed and quantitative immunohistochemistry. Nat. Protoc. 2007, 2, 1152-1165.

[8] Fountaine, T. J.; Wincovitch, S. M.; Geho, D. H.; Garfield, S. H.; Pittaluga, S. Multispectral imaging of clinically relevant cellular targets in tonsil and lymphoid tissue using semiconductor quantum dots. Modern Pathol. 2006, 19, 1181-1191.

[9] Nie, S. M.; Emery, S. R. Probing single molecules and single nanoparticles by surface-enhanced Raman scattering. Science 1997, 275, 1102-1106.

[10] Cao, Y. W. C.; Jin, R. C.; Mirkin, C. A. Nanoparticles with Raman spectroscopic fingerprints for DNA and RNA detection. Science 2002, 297, 1536-1540.

[11] Keren, S.; Zavaleta, C.; Cheng, Z.; de la Zerda, A.; Gheysens, O.; Gambhir, S. S. Noninvasive molecular imaging of small living subjects using Raman spectroscopy. Proc. Nat. Acad. Sci. USA 2008, 105, 5844-5849.

[12] Liu, Z.; Tabakman, S.; Welsher, K.; Dai, H. Carbon nanotubes in biology and medicine: In vitro and in vivo detection, imaging and drug delivery. Nano Res. 2009, 2, $85-120$.

[13] Liu, Z.; Cai, W.; He, L.; Nakayama, N.; Chen, K.; Sun, X.; Chen, X.; Dai, H. In vivo biodistribution and highly efficient tumour targeting of carbon nanotubes in mice. Nat Nanotechnol. 2007, 2, 47-52.

[14] Liu, Z.; Sun, X.; Nakayama, N.; Dai, H. Supramolecular chemistry on water-soluble carbon nanotubes for drug loading and delivery. ACS Nano 2007, 1, 50-56.

[15] Feazell, R. P.; Nakayama-Ratchford, N.; Dai, H.; Lippard, S.
J. Soluble single-walled carbon nanotubes as longboat delivery systems for platinum(IV) anticancer drug design. $J$. Am. Chem. Soc. 2007, 129, 8438-8439.

[16] Moon, H. K.; Chang, C. I.; Lee, D. -K.; Choi, H. C. Effect of nucleases on the cellular internalization of fluorescent labeled DNA-functionalized single-walled carbon nanotubes. Nano Res. 2008, 1, 351-360.

[17] Chen, R. J.; Bangsaruntip, S.; Drouvalakis, K. A.; Kam, N. W. S.; Shim, M.; Li, Y. M.; Kim, W.; Utz, P. J.; Dai, H. J. Noncovalent functionalization of carbon nanotubes for highly specific electronic biosensors. Proc. Nat. Acad. Sci. USA 2003, 100, 4984-4989.

[18] Chen, Z.; Tabakman, S. M.; Goodwin, A. P.; Kattah, M. G.; Daranciang, D.; Wang, X.; Zhang, G.; Li, X.; Liu, Z.; Utz, P. J. et al. Protein microarrays with carbon nanotubes as multicolor Raman labels. Nat. Biotechnol. 2008, 26, 1285-1292.

[19] Welsher, K.; Liu, Z.; D, D.; Dai, H. Selective probing and imaging of cells with single walled carbon nanotubes as near-infrared fluorescent molecules. Nano Lett. 2008, 8, 586-590.

[20] Cherukuri, P.; Gannon, C. J.; Leeuw, T. K.; Schmidt, H. K.; Smalley, R. E.; Curley, S. A.; Weisman, R. B. Mammalian pharmacokinetics of carbon nanotubes using intrinsic nearinfrared fluorescence. Proc. Natl. Acad. Sci. USA 2006, 103, 18882-18886.

[21] Hanlon, E. B.; Manoharan, R.; Koo, T. W.; Shafer, K. E.; Motz, J. T.; Fitzmaurice, M.; Kramer, J. R.; Itzkan, I.; Dasari, R. R.; Feld, M. S. Prospects for in vivo Raman spectroscopy. Phys. Med. Biol. 2000, 45, R1-R59.

[22] O'Connell, M. J.; Bachilo, S. M.; Huffman, C. B.; Moore, V. C.; Strano, M. S.; Haroz, E. H.; Rialon, K. L.; Boul, P. J.; Noon, W. H.; Kittrell, C. et al. Band gap fluorescence from individual single-walled carbon nanotubes. Science 2002, 297, 593-596.

[23] Rao, A. M.; Richter, E.; Bandow, S.; Chase, B.; Eklund, P. C.; Williams, K. A.; Fang, S.; Subbaswamy, K. R.; Menon, M.; Thess, A. et al. Diameter-selective Raman scattering from vibrational modes in carbon nanotubes. Science 1997, $275,187-191$

[24] Liu, Z.; Li, X.; Tabakman, S. M.; Jiang, K.; Fan, S.; Dai, H. Multiplexed multi-color Raman imaging of live cells with isotopically modified single walled carbon nanotubes. J. Am. Chem. Soc. 2008, 130, 13540-13541.

[25] Li, X. L.; Tu, X. M.; Zaric, S.; Welsher, K.; Seo, W. S.; Zhao, W.; Dai, H. J. Selective synthesis combined with chemical separation of single-walled carbon nanotubes for chirality selection. J. Am. Chem. Soc. 2007, 129, 15770-15771.

[26] Liu, Z.; Davis, C.; Cai, W.; He, L.; Chen, X.; Dai, H. 
Circulation and long-term fate of functionalized, biocompatible single-walled carbon nanotubes in mice probed by Raman spectroscopy. Proc. Natl. Acad. Sci. USA 2008, 105, 1410-1415.

[27] Liu, Z.; Cai, W. B.; He, L. N.; Nakayama, N.; Chen, K.; Sun, X. M.; Chen, X. Y.; Dai, H. J. In vivo biodistribution and highly efficient tumour targeting of carbon nanotubes in mice. Nat. Nanotechnol. 2007, 2, 47-52.

[28] Liu, Z.; Tabakman, S. M.; Chen, Z.; Dai, H. Preparation of carbon nanotube bioconjugates for biomedical applications. Nat. Protoc. 2009, 4, 1372-1382.

[29] Lewis, G. D.; Figari, I.; Fendly, B.; Wong, W. L.; Carter, P.; Gorman, C.; Shepard, H. M. Differential responses of human tumor cell lines to anti-p185 ${ }^{\mathrm{HER} 2}$ monoclonal antibodies. Cancer Immunol. Immun. 1993, 37, 255-263.

[30] Haubner, R.; Wester, H. -J.; Weber, W. A.; Mang, C.; Ziegler, S. I.; Goodman, S. L.; Senekowitsch-Schmidtke, R.; Kessler, H.; Schwaiger, M. Noninvasive imaging of $\alpha v \beta 3$ integrin expression using 18F-labeled RGD-containing glycopeptide and positron emission tomography. Cancer Res. 2001, 61, 1781-1785.

[31] Cai, W.; Shin, D. -W.; Chen, K.; Gheysens, O.; Cao, Q.; Wang, S. X.; Gambhir, S. S.; Chen, X. Peptide-labeled near-infrared quantum dots for imaging tumor vasculature in living subjects. Nano Lett. 2006, 6, 669-676.

[32] Eliceiri, B. P.; Cheresh, D. A. The role of $\alpha \mathrm{v}$ integrins during angiogenesis: Insights into potential mechanisms of action and clinical development. J. Clin. Invest. 1999, 103, 1227-1230.

[33] Mulder, W. J. M.; Strijkers, G. J.; Habets, J. W.; Bleeker, E. J. W.; van der Schaft, D. W. J.; Storm, G.; Koning, G. A.; Griffioen, A. W.; Nicolay, K. MR molecular imaging and fluorescence microscopy for identification of activated tumor endothelium using a bimodal lipidic nanoparticle. FASEB J. 2005, 19, 2008-2010.

[34] Bonner, J. A.; Buchsbaum, D. J.; Russo, S. M.; Fiveash, J. B.; Trummell, H. Q.; Curiel, D. T.; Raisch, K. P. AntiEGFR-mediated radiosensitization as a result of augmented EGFR expression. Int. J. Radiat. Oncol. 2004, 59, 2-10.

[35] Milenic, D. E.; Wong, K. J.; Baidoo, K. E.; Ray, G. L.; Garmestani, K.; Williams, M.; Brechbiel, M. W. Cetuximab: Preclinical evaluation of a monoclonal antibody targeting EGFR for radioimmunodiagnostic and radioimmunotherapeutic applications. Cancer Biother. Radio. 2008, 23, 619-631.

[36] Milenic, D. E.; Garmestani, K.; Brady, E. D.; Albert, P. S.; Ma, D.; Abdulla, A.; Brechbiel, M. W. Targeting of HER2 antigen for the treatment of disseminated peritoneal disease. Clin. Cancer Res. 2004, 10, 7834-7841.

[37] Oliveira, S.; Henegouwen, P. M. V. E.; Storm, G.; Schiffelers, R. M. Molecular biology of epidermal growth factor receptor inhibition for cancer therapy. Expert Opin. Biol. Th. 2006, 6, 605-617.

[38] Heller, D. A.; Baik, S.; Eurell, T. E.; Strano, M. S. Singlewalled carbon nanotube spectroscopy in live cells: Towards long-term labels and optical sensors. Adv. Mater. 2005, 17, 2793-2799.

[39] Freudiger, C. W.; Min, W.; Saar, B. G.; Lu, S.; Holtom, G. R.; He, C.; Tsai, J. C.; Kang, J. X.; Xie, X. S. Label-free biomedical imaging with high sensitivity by stimulated Raman scattering microscopy. Science 2008, 322, 1857-1861.

[40] Tu, X.; Zheng, M. A DNA-based approach to the carbon nanotube sorting problem. Nano Res. 2008, 1, 185-194.

[41] Kam, N. W. S.; O'Connell, M.; Wisdom, J. A.; Dai, H. Carbon nanotubes as multifunctional biological transporters and near-infrared agents for selective cancer cell destruction. Proc. Natl. Acad. Sci. USA 2005, 102, 11600-11605. 